\title{
COMMON DOS ERROR MESSAGES
}

Bad command or file name

- DOS cannot find the file you have named

Access denied/attempted write-protect violation

- you are attempting to write to a write-protected disk

BDOS Function $=x x$ File $=$ filename

- defective or unformatted disk

Disk Read/Write error

- corrupt disk

ERROR - Diskette is full

- insufficient space to save your file

No room in directory for file

- the directory is full (a directory can only store a finite num-

ber of entries, even though the disk itself has space)

Invalid parameter

- DOS cannot understand part of the command

File not found

- DOS cannot locate the file you have specified

Syntax error

- DOS cannot understand your command 